\title{
Modelagem Geofísica na área da mina Cerro Rico em Lavras do Sul (RS), a partir da integração de dados de resistividade elétrica e de cargabilidade (IP).
}

Rosales, M. J. T., UNIPAMPA, Alcalde G. ${ }^{*}$, UNIPAMPA, Ramos, W. A., UNIPAMPA.

Copyright 2019, SBGf - Sociedade Brasileira de Geofísica

This paper was prepared for presentation during the $16^{\text {th }}$ International Congress of the Brazilian Geophysical Society held in Rio de Janeiro, Brazil, 19-22 August 2019.

Contents of this paper were reviewed by the Technical Committee of the $16^{\text {th }}$ International Congress of the Brazilian Geophysical Society and do not necessarily represent any position of the SBGf, its officers or members. Electronic reproduction or storage of any part of this paper for commercial purposes without the written consent of the Brazilian Geophysical Society is prohibited.

\section{Abstract}

The research area covers the Cerro Rico Mine and is located $7 \mathrm{~km}$ east of the city of Lavras do Sul, and is bounded by the geographical coordinates: Lengths: 53, 81200 W - 53.83400 W and Latitudes: 30, 81100 S$30.81870 \mathrm{~S}$. The mineral occurrence of $\mathrm{Au}, \mathrm{Ag}$ and copper $(\mathrm{Cu})$, is related to the presence of zones of hydrothermal alteration and is conditioned the existence of quartz filaments that present preferential directions northwest (NW) and east - west ( $E-W)$, present in the rocks characterized as tuffs, andesites, rhyolitic spills and volcanic conglomerates of the Hilário Formation (604 $590 \mathrm{Ma}$ ), belonging to the Bom Jardim Group, or Alogrupo Bom Jardim. The main objective of this work is to contribute to the clarification of the local geology framework by integrating and interpreting aerial and terrestrial geophysical data in a detailed scale, in order to identify and characterize areas of hydrothermal alteration associated with zones of fault and / or fractures possibly related to mineralization of base metals $(\mathrm{Ag}$ and $\mathrm{Cu})$ and gold $(\mathrm{Au})$. The aerogiophysical data and the lithological descriptions of the drilling holes were provided by Amarillo Mineração do Brasil Ltda, belonging to the gamma - spectrometric aerogeophysical survey - Lavras do Sul Project - Rio Grande do Sul, 2007. The Electrical Resistivity and Induced Polarization (IP) data were acquired using the Electrical Walk (Diplo-Dipole Arrangement) technique and the Vertical Electrical Probing (Wenner Arrangement) technique, along two regional geophysical profiles, in two directions Preferences: SW - NE, and NW - SE. The presence of chargeability anomalies with values of 10 to $14 \mathrm{mV} / \mathrm{V}$ at depths of approximately 25-30 meters suggests that the presence of metallic mineralization, ie the presence of metallic oxides, is associated with failure zones and / or fractures that present continuity in depth, being able to be affecting the andesitic base. The results of the interpretation of geophysical-geological models for Profile A-A 'and Profile B-B', indicated estimates of the depth of the top of the andesitic basement around 25 to $35 \mathrm{~m}$, characterized by the presence of irregular, probably affected by fractures and / or failures.

\section{Introdução}

A área da pesquisa abrange a Mina Cerro Rico e localizase a $7 \mathrm{~km}$ ao leste da cidade de Lavras do Sul, e delimitase pelas coordenadas geográficas: Longitudes: 53,81200 W - 53,83400 W e as Latitudes: $30,81100 \mathrm{~S}-30,81870 \mathrm{~S}$. A ocorrência mineral de $\mathrm{Au}, \mathrm{Ag}$ e cobre $(\mathrm{Cu})$, encontra-se condicionada à existência de filões de quartzo que apresentam direções preferenciais noroeste (NW) e lesteoeste $(\mathrm{E}-\mathrm{W})$, presentes nas rochas caracterizadas como tufos, andesitos, derrames riolíticos e conglomerados vulcânicos da Formação Hilário (604 - 590 Ma), pertencentes ao Grupo Bom Jardim, ou Alogrupo Bom Jardim. A geologia local da área se caracteriza pela presença de rochas pertencentes à Formação Hilário que são descritas como piroclásticas, tufos, efusivas (subvulcânicas) e conglomerados vulcânicos (GASTAL et al. 2015).

As porções com mineralizações na região da Mina Cerro Rico se encontram predominantemente em tufos, os quais sofreram alteração hidrotermal devido a fluidos vindos das porções intrusivas do Complexo Intrusivo Lavras do Sul e águas meteóricas. Segundo Bongiolo (2006), estas alterações desencadearam principalmente porções mineralizadas e alteradas relacionadas a metais como Au e Cu.

\section{Materiais e Métodos}

O arcabouço geológico da área de estudo se ilustra na Figura 1, ressalta-se a área demarcada pela presença dos perfis geofísicos A - A' ( NW - SE) e B - B' (SW $\mathrm{NE}$ ), a presença de rochas vulcânicas e subvulcânicas pertencentes a formação Hilário (604 - 590 Ma), pertencente ao Grupo Bom Jardim (GASTAL et al. 2015). 


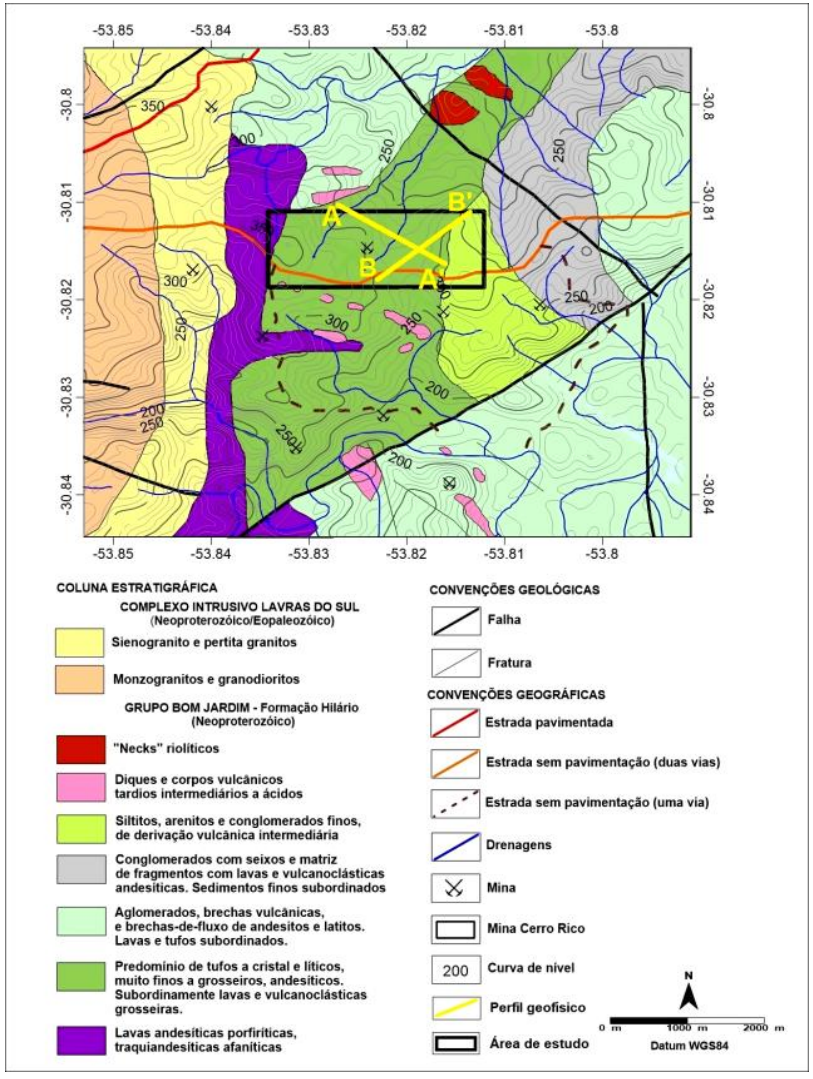

Figura 1 - Mapa geológico da área de estudo (Fonte: Adaptado de Minerar, 1998.)

Os dados aerogeofísicos e as descrições litológicas dos furos de sondagem utilizados na pesquisa foram gentilmente cedidos pela empresa Amarillo Mineração do Brasil Ltda, pertencentes ao Levantamento aerogeofísico gamaespectrométrico - Projeto Lavras do Sul - Rio Grande do Sul, o qual foi executado no ano 2007 pela empresa Prospectors Aerolevantamentos e Sistemas Ltda.

O processamento dos dados aerogamaespectrométricos seguiu uma metodologia convencional para a prospecção mineral, onde foram confeccionados mapas de imagens dos valores anômalos das concentrações dos radioelementos, destacando os mais relevantes: mapa do Fator $\mathrm{F},(\mathrm{F}=\mathrm{K}(\mathrm{eU} / \mathrm{eTh}))$, mapa do potássio anômalo (Kd), mapa do urânio anômalo (Ud) e mapa ternário de composição colorida RGB dos parâmetros Kd, Ud e Fator $\mathrm{F}$.

Segundo Efimov (1978), a anomalia do Fator F constitui uma ferramenta como subsídio no reconhecimento de depósitos minerais associados ao hidrotermalismo. O Fator $\mathrm{F}$ pode ressaltar o enriquecimento de potássio em relação à razão entre o urânio e tório e destacar a presença de processos de alteração hidrotermal associados à mineralização.

As anômalias de potássio anômalo (Kd) e de urânio anômalo (Ud) são fortes indicadores de áreas com enriquecimento de potássio e de urânio associados a processos hidrotermais (Pires, 1995).
Os dados de Polarização Induzida (IP) foram adquiridos com a técnica de Caminhamento Elétrico (Arranjo DipoloDipolo) e com a técnica de Sondagem Elétrica Vertical (Arranjo Wenner), ao longo de dois perfis geofísicos regionais, segundo duas direções preferenciais: Perfil B $B^{\prime}(S W-N E)$, e Perfil A - A' (NW - SE). Como resultado da integração e interpretação dos dados gamaespectrométricos, dados de resistividade e de polarização induzida foram confeccionados dois modelos geofísicos-geológicos 2D, que relacionam 0 comportamento das anomalias do Fator $\mathrm{F}$, do potássio anômalo (Kd) e do urânio anômalo (Ud), com as anomalias de cargabilidade (IP).

\section{Resultados}

$\mathrm{Na}$ porção central da área de estudo conforme os gráficos das anomalias de Ud, Kd e Fator $\mathrm{F}$ (Figura 2); para o perfil geofísico A - A' (NW-SE), se observa um patamar significativo no comportamento das anomalias de Fator F, Potássio anômalo e Urânio anômalo, isto é: intervalo de valores para $\circ$ Fator $F$ de 1,0 a 1,04; intervalo de valores para o $\mathrm{Kd}$ de 0,44 a 0,46 e intervalo de valores para o Ud de 0,52 a 0,54 . Para o perfil geofísico B - B' (SW-NE), observa-se um patamar de menor amplitude das anomalias, se comparado com o perfil A - $A^{\prime}$, isto é: intervalo de valores para o Fator $F$ de 0,70 a 0,78 ; intervalo de valores para $0 \mathrm{Kd}$ de aproximadamente 0,1 e intervalo de valores para o Ud de 0,1 a 0,2 . Este comportamento se deve ao fato do perfil geofísico B - B' (SW - NE) estar posicionado na borda sudeste das anomalias de Fator F, Kd e Ud (Figura 4). O posicionamento destes perfis em campo obedeceu a um critério de interpretação geofísico-geológica, por estarem próximos aos furos de sondagem LDH-188 e LDH-189 (Figura 3) de maneira a correlacionar as litologias presentes no furo com os modelos geológico-geofísicos propostos para os perfis A - A' e B - B'. Conforme se observa na figura 4 no mapa ternário de composição colorida RGB dos parâmetros Kd, Ud e Fator F, sugerese na presença de anomalias caracterizadas pela cor branca, altos valores de Fator $F, K d$ e Ud associados a processos de hidrotermalismo, os quais se caracterizam pelo enriquecimento destes dois radioelementos. 


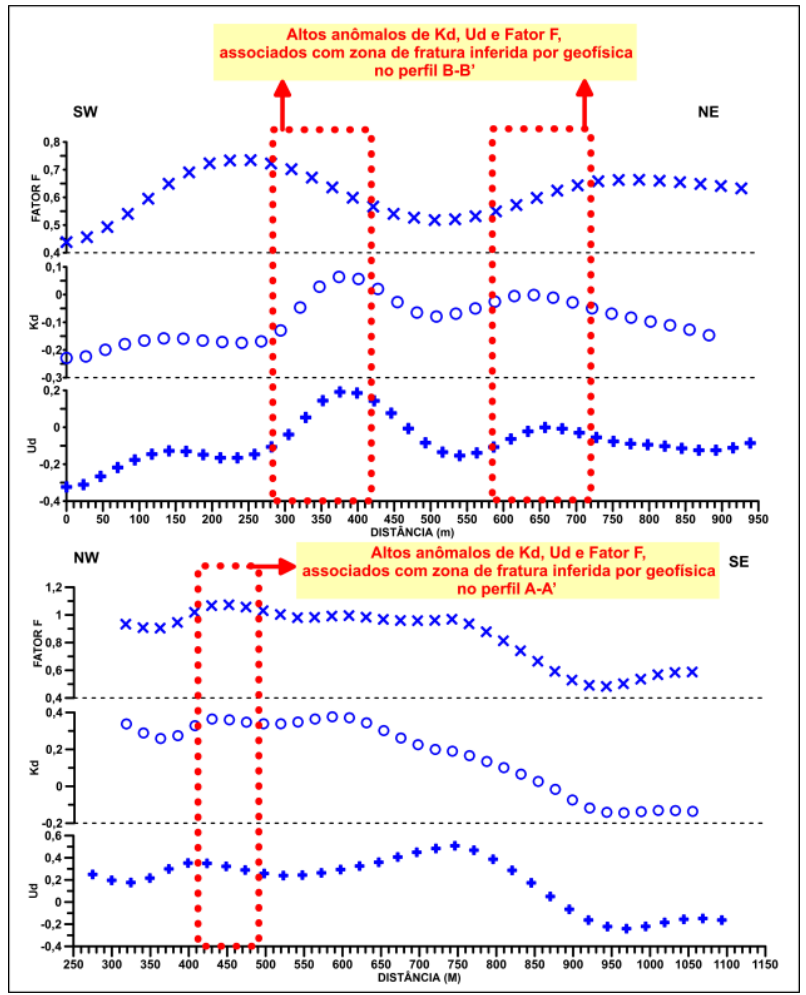

Figura 2 - Esquema gráfico das anomalias de Ud, Kde Fator $F$ nos perfis geofísicos $A-A^{\prime}(N W-S E)$ e $B-B^{\prime}$ (SW-NE).

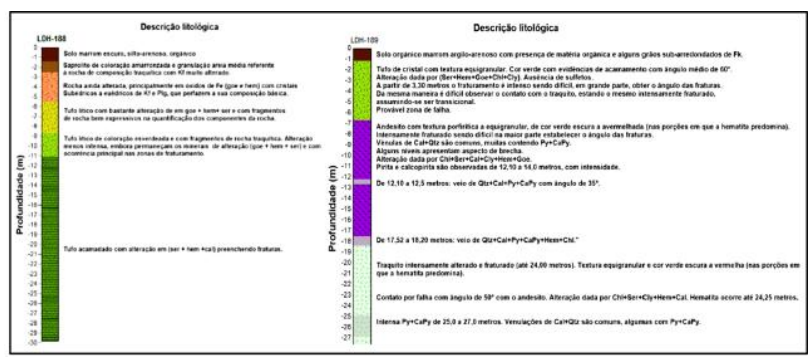

Figura 3 - Colunas litológicas dos furos de sondagem LDH-188 e LDH-189 (Fonte: Amarillo, 2007.).

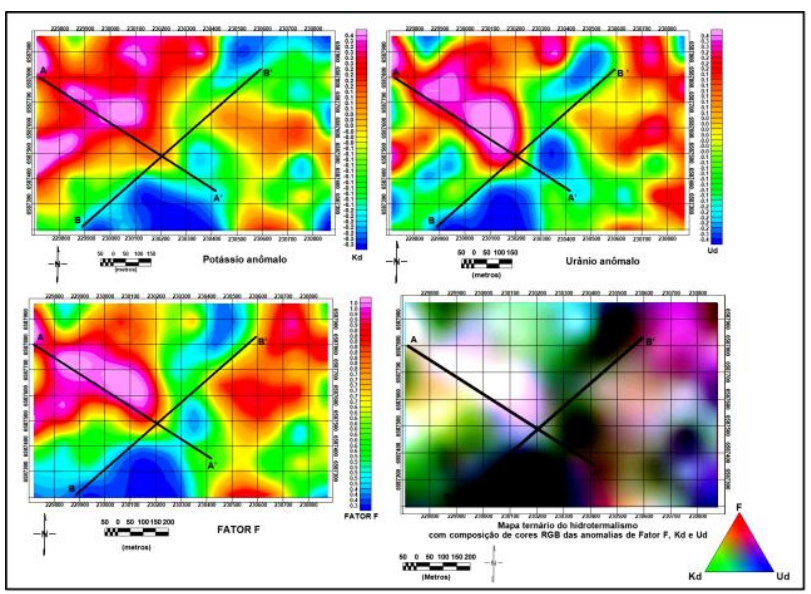

Figura 4 - Mapas de imagem das anomalias de Kd, Ud, Fator $F$ e mapa ternário com composição colorida $R G B$ (Kd,Ud e Fator F).
Nos modelos 2D de dados de IP para os perfis A-A' e BB' observam-se anomalias de cargabilidade com valores de 10 a $14 \mathrm{mV} / \mathrm{V}$ (Figura 5), em profundidades aproximadas de 25 - 30 metros, o que sugere-se que estejam associadas a presença de mineralização metálica, isto é presença de óxidos metálicos, vinculada com zonas de falhas e/ou fraturas que apresentem continuidade em profundidade, podendo estar afetando o embasamento andesítico (Figura 6).

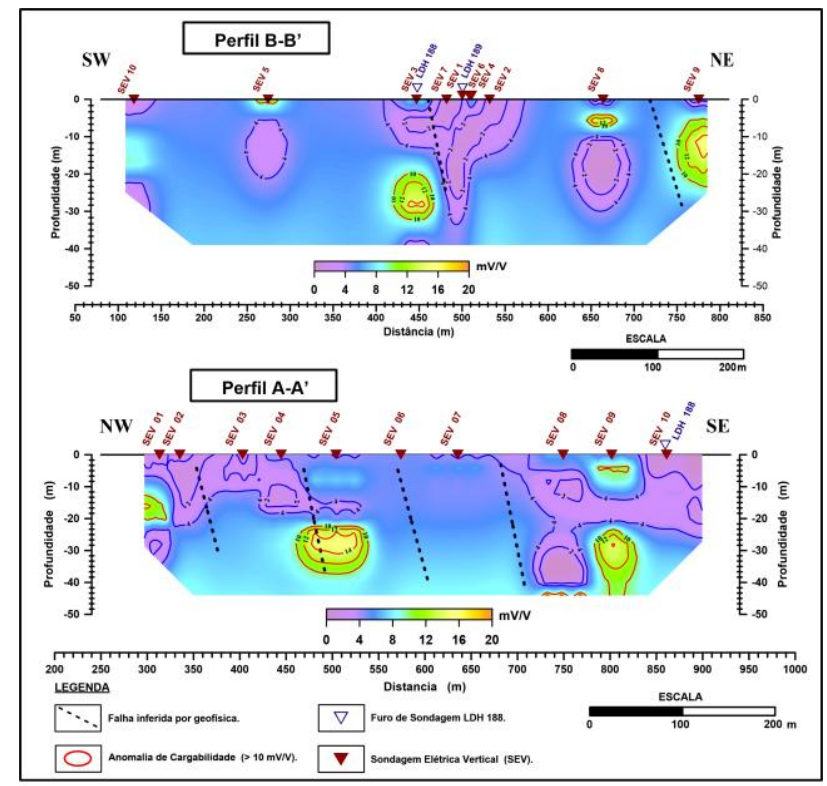

Figura 5-Modelo 2D de cargabilidade para os perfis $A$ $A^{\prime}(N W-S E)$ e $B$ - B' (SW-NE).

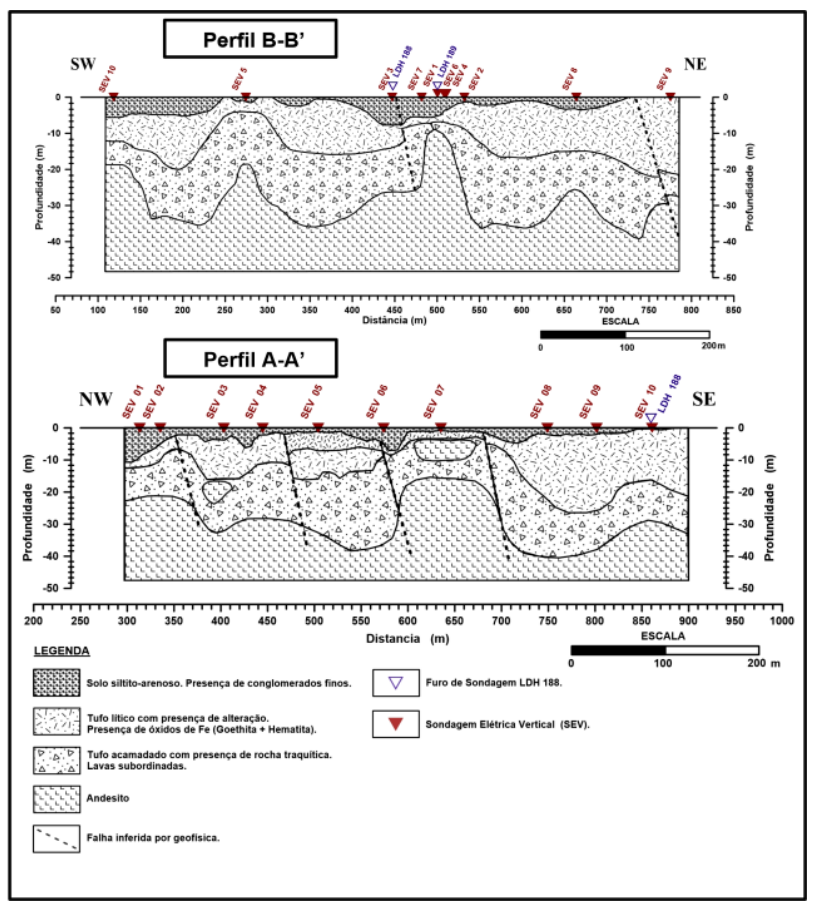

Figura 6 - Modelos geológicos para os perfis $A$ - A' (NW$S E)$ e $B-B^{\prime}(S W-N E)$. 


\section{Conclusões}

Os altos valores de Fator F, Kd, e do Ud, para os dois perfis sugerem um enriquecimento de potássio e urânio nestes setores, podendo estar associados a provável presença de processos hidrotermais, o enriquecimento dos teores do $\mathrm{K}$ e do $\mathrm{U}$, encontram-se diretamente associados aos processos de sericitização, cloritização e potassificação, que acompanham o hidrotermalismo na gênese dos depósitos metálicos, estes processos podem estar sendo favorecidos pela presença de água em zonas de fraturas e/ou falhas, inferidas pela geofísica. Conforme os modelos geológicos 2D para o Perfil A - A' e Perfil B B', para a área da Mina Cerro Rico, pode-se inferir segundo critérios interpretativos, que o topo do embasamento andesítico se encontra a uma profundidade de aproximadamente 25 a $35 \mathrm{~m}$, caracterizado pela presença de um relevo irregular, muito provavelmente afetado por fraturas e/ou falhas. Camadas de solo com fragmentos de rochas vulcânicas, e/ou a presença de tufos com lavas subordinadas, caracterizam um intervalo de profundidades entre $5 \mathrm{a} 25 \mathrm{~m}$. é notório ressaltar que a interpretação dos modelos geológicos (Perfil A - A' e Perfil B - B') foi baseada na correlação com a descrição das litologias dos furos de sondagem LDH-188 e LDH-189. A provável presença de fraturas preenchidas por veios de quartzo, com possível presença de mineralização metálica, caracteriza alguns setores da área com anomalias de cargabilidade (IP) com valores em torno de 12 - $14 \mathrm{mv} / \mathrm{V}$, resultando em alvos potenciais atrativos, para dar continuidade e orientar futuras pesquisas relacionadas à prospecção aurífera na Mina Cerro Rico.

\section{Agradecimentos}

Agradecemos a empresa Amarillo Mineração do Brasil Ltda, por ter cedido gentilmente os dados aereogeofísicos pertencentes ao Levantamento aerogeofísico gamaespectrométrico - Projeto Lavras do Sul - Rio Grande do Sul, assim como a informação geológica referente aos furos de sondagem, e por ter permitido o acesso físico à área da pesquisa para a execução dos levantamentos geofísicos terrestres.

\section{Referências}

BONGIOLO, E.M. INTEGRAÇÃO DE DADOS MINERALÓGICOS, ISÓTOPOS ESTÁVEIS (O, H) E POROSIDADE DE ROCHAS (14C-PMMA) NO RECONHECIMENTO DA EVOLUÇÃO DA ALTERAÇÃO NO SISTEMA HIDROTERMAL DE LAVRAS DO SUL/RS, BRASIL. Tese de doutorado, Universidade Federal do Rio Grande do Sul. 2006.

EFIMOV, A. V. MULTIPLIKATIVNYJ POKAZATEL DLJA VYDELENIJA ENDOGENNYCH RUD PÓ AEROGAMMASPEKTROMETRICESKIM DANNYM. In: Metody rudnoj geofiziki, edited by: Naucnoproizvodstvennoje objedinenie "Geofizika" Leningrad. 1978.
GASTAL, M. C.; FERREIRA, F. J. F.; CUNHA, J. U.; ESMERIS, C.; KOESTER, E.; RAPOSO, M. I. B.; ROSSETTI, M. M. M. ALOJAMENTO DO GRANITO LAVRAS E A MINERALIZAÇÃO AURÍFERA DURANTE EVOLUÇÃO DE CENTRO VULCANO-PLUTÔNICO PÓS-COLISIONAL, OESTE DO ESCUDO SULRIOGRANDENSE: DADOS GEOFÍSICOS E ESTRUTURAIS. Instituto de Geociências, Universidade Federal do Rio Grande do Sul. 2015.

MINERAR. MAPA GEOLÓGICO DA MINA CERRO RICO. Projeto de Diagnóstico do Potencial Mineral do Município de Lavras do Sul. 1998.

MIRANDA, R. R. Prospectors Aerolevantamentos e Sitemas Ltda. LEVANTAMENTO AEROGEOFÍSICO GRADIOMÉTRICO E GAMAESPECTROMÉTRICO. Projeto Lavras do Sul, Rio Grande do Sul. 2007.

PIRES, A. C. B. IDENTIFICAÇÃO GEOFÍSICA DE ÁREAS DE ALTERAÇÃO HIDROTERMAL, CRIXÁSGUARINOS, Goiás. Revista Brasileira de Geociências, 25(1): 61-68 p.1995. 